\title{
Fixed point theorem for reflexive Banach spaces and uniformly convex non positively curved metric spaces
}

\author{
Izhar Oppenheim \\ Department of Mathematics \\ The Ohio State University \\ Columbus, OH 43210, USA \\ E-mail: izharo@gmail.com
}

December 29, 2017

\begin{abstract}
This article generalizes the work of Ballmann and Światkowski to the case of Reflexive Banach spaces and uniformly convex Busemann spaces, thus giving a new fixed point criterion for groups acting on simplicial complexes.
\end{abstract}

Mathematics Subject Classification (2010). Primary 20F65

Keywords. Fixed point property, Banach space, Busemann space, simplicial complex, Laplacian

\section{Introduction}

For a finite graph $(V, E)$ the Laplacian is a positive operator defined on functions $f: V \rightarrow \mathbb{R}$. One can generalize the definition of the Laplacian for a simplicial complex $X$ of any dimension and for such a complex the Laplacian is again a positive operator.

Ballmann and Światkowski in BŚ97 and independently Żuk's in Żuk96. used the geometric information given by the Laplacian eigenvalues to give criteria for the vanishing of cohomologies of a group $\Gamma$ acting on a simplicial complex. The most famous result of this type is the Żuk criterion which states that a group acting geometrically (i.e. cocompactly and proper discontinuously) on a 2-dimensional simplicial complex has property $(\mathrm{T})$ if the smallest positive Laplacian eigenvalues at the link of every vertex is large enough. It is well known 
that in the above setting, property $(\mathrm{T})$ is equivalent to a fixed point property for action by isometries on a Real Hilbert space (see for instance [BdlHV08]).

In this article, we generalize the Żuk criterion to reflexive Banach spaces and uniformly convex non positively curved Busemann spaces and get a fixed point criterion for those spaces relaying on the geometry of the links of vertices. The method that we use is basically taken from Gromov in Gro03 (3.11), but we improve it so it doesn't require any scaling limit arguments (and generalize the form of the energy function).

Structure of the paper. The first section gathers needed results about groups acting on simplicial complexes, Uniformly convex Busemann non positively curved spaces and Reflexive Banach spaces. The second section contains the main theorem and its proof.

\section{Framework and Preliminaries}

\subsection{General Settings}

Throughout this paper $X$ is a simplicial complex of dimension $n \geq 2$ such that all the links of $X$ are connected and we assume that the links of all the vertices of $X$ are finite. Also $\Gamma$ is a locally compact, properly discontinuous, unimodular group of automorphisms of $X$ acting cocompactly on $X$.

Following [BŚ97] we introduce the following notations:

1. For $0 \leq k \leq n$, denote by $\Sigma(k)$ the set of ordered $k$-simplices (i.e. $\sigma \in \Sigma(k)$ is and ordered $k+1$-tuple of vertices) and choose a set $\Sigma(k, \Gamma) \subseteq \Sigma(k)$ of representatives of $\Gamma$-orbits.

2. For a simplex $\sigma \in \Sigma(k)$, denote by $\Gamma_{\sigma}$ the stabilizer of $\sigma$ and by $\left|\Gamma_{\sigma}\right|$ the measure of $\Gamma_{\sigma}$ with respect to the Haar measure.

The following proposition is taken from BŚ97, DJ00:

Proposition 2.1. BŚ97, Lemma 1.3], [DJ00, Lemma 3.3] For $0 \leq l<k \leq n$, let $f=f(\tau, \sigma)$ be a $\Gamma$-invariant function on the set of pairs $(\tau, \sigma)$, where $\tau$ is an ordered $l$-simplex and $\sigma$ is an ordered $k$-simplex with $\tau \subset \sigma$ Then

$$
\sum_{\sigma \in \Sigma(k, \Gamma)} \sum_{\substack{\tau \in \Sigma(l) \\ \tau \subset \sigma}} \frac{f(\tau, \sigma)}{\left|\Gamma_{\sigma}\right|}=\sum_{\tau \in \Sigma(l, \Gamma)} \sum_{\substack{\sigma \in \Sigma(k) \\ \tau \subset \sigma}} \frac{f(\tau, \sigma)}{\left|\Gamma_{\tau}\right|}
$$

The reader should note, that from now on we will use the above proposition to change the order of summation without mentioning it explicitly.

Definition 2.2. A weight on $X$ is an equivariant function $m: \bigcup_{2 \geq k \geq 1} \Sigma(k) \rightarrow$ $\mathbb{R}_{>0}^{+}$such that: 
1. For every $\tau=\left(v_{0}, \ldots, v_{k}\right)$ and for every permutation $\sigma \in S_{k}$ we have $m\left(\left(v_{0}, \ldots, v_{k}\right)\right)=m\left(\left(v_{\sigma(0)}, \ldots, v_{\sigma(k)}\right)\right.$.

2. There is a $C(m)$ such that for every $\tau \in \Sigma(1)$ we have the following equality

$$
\sum_{\sigma \in \Sigma(2), \tau \subset \sigma} m(\sigma)=3 ! C(m) m(\tau)
$$

Where $\tau \subset \sigma$ means that all the vertices of $\tau$ are contained in $\sigma$ (with no regard to the ordering).

Example 2.3. In BŚ the function $m$ was defined as: for every $\tau \in \Sigma(k)$, $m(\tau)$ is the number of (unordered) simplices of dimension $n$ that contain $\tau$. In that case, $C(m)=n-1$.

Remark 2.4. There is a lot of freedom in our definition of the weight function. Without loss of generality, one can always normalize the weight function such that $C(m)=1$. It is obvious that in the normalized case the function $m$ is determined by its values on $\Sigma(2)$. We chose not to normalize the weight function in this paper as a matter of convenience and so that the reader could easily compare our results to those proven in [BŚ97].

Definition 2.5. Let $u \in \Sigma(0)$, denote by $X_{u}$ the link of $u$ in $X$, that is, the subcomplex of dimension $n-1$ consisting on simplices $\sigma=\left(w_{0}, \ldots, w_{k}\right)$ such that $\{u\},\left\{w_{0}, \ldots, w_{k}\right\}$ are disjoint as sets and $\left(u, w_{0}, \ldots, w_{k}\right)=u \sigma \in \Sigma(k+1)$. As stated above, $X$ is locally finite which means that $X_{u}$ is a finite simplicial complex.

1. For $0 \leq k \leq n-1$, denote by $\Sigma_{u}(k)$ the set of ordered $k$-simplices.

2. For a simplex $\sigma \in \Sigma_{u}(k)$ denote by $m_{u}(\sigma)=m(u \sigma)$.

\subsection{Uniformly convex Busemann non positively curved spaces}

In this subsection we will give definitions and some results about uniformly convex Busemann non positively curved spaces spaces.

Let $(Z, d)$ be a unique geodesic complete metric space, i.e. between any two points $x, y \in Z$ there is a unique geodesic connecting $x$ and $y$. For $x, y \in Z$ and $0 \leq t \leq 1$ denote by $t x+(1-t) y$ the point on the geodesic connecting $x$ and $y$ such that

$$
d(x, t x+(1-t) y)=t d(x, y), d(y, t x+(1-t) y)=(1-t) d(x, y)
$$

This is of course only a notation because $Z$ need not be a vector space. 
Definition 2.6. A uniquely geodesic metri 1 space $(Z, d)$ will be called uniformly convex if there is a map $\theta:(0, \infty) \times(0,2] \rightarrow(0,1]$ such that for every $r>0$ and every $\varepsilon \in(0,2]$, for every three points $a, x, y \in Z$ the following implication holds:

$$
\begin{aligned}
d(a, x) & \leq r \\
d(a, y) & \leq r \\
d(x, y) & \geq \varepsilon r
\end{aligned} \quad \Rightarrow d\left(\frac{1}{2} x+\frac{1}{2} y, a\right) \leq(1-\theta(r, \varepsilon)) r
$$

and

$$
\forall \varepsilon \in(0,2], \theta(\varepsilon)=\inf \{\theta(r, \varepsilon): r>0\}>0
$$

The map $\theta$ is called the modulus of uniform convexity.

Remark 2.7. The above definition is closely related to [GR84][page 107] (it appears also in [RS90] and GKM08]). The reader should note there are other (non equivalent) definitions for uniformly convex metric space - see for instance [KL10].

Examples of uniformly convex metric spaces:

1. Hilbert spaces.

2. $L^{p}$ spaces for $1<p<\infty$.

3. $C A T(0)$ spaces - the modulus of convexity might depend on $r$ but it is bounded by the modulus of convexity of a Hilbert space (for the same $\varepsilon$ ).

The following proposition is stated and proven in GR84 for the Banach case (see GR84][Theorem 2.1], and it is also mentioned there that the proof is the same for the genera case of a uniformly convex metric space (see GR84][Theorem 18.1]). We shall repeat the proof here for completeness.

Proposition 2.8. Let $(Z, d)$ be a complete uniquely geodesic uniformly convex metric space, then for closed convex bounded non empty sets $C_{n} \subset Z$ such that $C_{n+1} \subset C_{n}$, we have $\bigcap_{n=1}^{\infty} C_{n} \neq \emptyset$.

Proof. Take arbitrary $x \in Z$. If $x \in \bigcap_{n=1}^{\infty} C_{n}$ we are done. If not there is some $N$, such that $d\left(x, C_{N}\right)>0$ (recall that $C_{N}$ is closed). Denote $r_{n}=$ $d\left(x, C_{n}\right)$, then $\left\{r_{n}\right\}$ is an increasing non negative sequence which is bounded from above because $C_{1}$ is bounded. Denote $r=\lim r_{n} \geq r_{N}>0$. Define $D_{n}=C_{n} \cap \bar{B}\left(x, r+\frac{1}{n}\right)$, by completeness it is enough to show that $\operatorname{diam}\left(D_{n}\right) \rightarrow 0$, because then $\bigcap_{n=1}^{\infty} C_{n} \supseteq \bigcap_{n=1}^{\infty} D_{n} \neq \emptyset$. Assume toward contradiction that $\operatorname{diam}\left(D_{n}\right) \rightarrow d>0$, then there is some $n_{0}$ such that for every $n>n_{0}$ we have $\frac{1}{n}<\frac{d}{2}$. For every $n>n_{0}$ we have points $x_{n}, y_{n} \in D_{n}$ such that

$$
d\left(x_{n}, y_{n}\right)>d-\frac{1}{n}>\frac{d}{2}=\frac{d}{2\left(r+\frac{1}{n}\right)}\left(r+\frac{1}{n}\right) \geq \frac{d}{2(r+1)}\left(r+\frac{1}{n}\right)
$$

\footnotetext{
${ }^{1}$ Uniquely geodesic means that every two points have a unique geodesic connecting them. From now on we will assume that our spaces are always uniquely geodesic.
} 
and since $x_{n}, y_{n} \in D_{n}$ we have $d\left(x, x_{n}\right), d\left(x, y_{n}\right) \leq r+\frac{1}{n}$. Note that $\frac{d}{2} \leq r+1$ because $D_{n} \subseteq \bar{B}(x, r+1)$ and therefore $\frac{d}{2(r+1)} \leq 1$. By uniform convexity, for every $n>n_{0}$ we have

$$
\begin{aligned}
r_{n} \leq d\left(x, \frac{1}{2} x_{n}+\right. & \left.\frac{1}{2} y_{n}\right) \leq\left(1-\theta\left(r+\frac{1}{n}, \frac{d}{2(r+1)}\right)\right)\left(r+\frac{1}{n}\right) \leq \\
& \leq\left(1-\theta\left(\frac{d}{2(r+1)}\right)\right)\left(r+\frac{1}{n}\right)
\end{aligned}
$$

we can take $n \rightarrow \infty$ and get that

$$
r \leq\left(1-\theta\left(\frac{d}{2(r+1)}\right)\right) r<r
$$

which is a contradiction.

The above proposition has a useful corollary (also taken from GR84][Proposition 18.2] with minor adaptations) :

Corollary 2.9. Let $(Z, d)$ be a complete uniquely geodesic uniformly convex metric space and let $f: Z \rightarrow \mathbb{R}^{+}$be a quasi-convex function, i.e.

$$
\forall 0 \leq t \leq 1, \forall x, y \in Z, f(t x+(1-t) y) \leq \max \{f(x), f(y)\}
$$

If there is a $c \in \mathbb{R}^{+}$such that the set $\{x: f(x) \leq c\}$ is non empty and bounded, then $f$ has a minimum. Moreover, if $f$ is strictly quasi-convex, i.e.

$$
\forall x, y \in Z, x \neq y, f\left(\frac{1}{2} x+\frac{1}{2} y\right)<\max \{f(x), f(y)\}
$$

then this minimum is unique.

Proof. Denote $c^{\prime}=\inf \{f(x): x \in Z\} \geq 0$, we shall show that $c^{\prime}$ is the minimum of $f$. If $c^{\prime}=c$ we are done because we know that $\{x: f(x) \leq c\}$ is non empty. Otherwise, there is an integer $n_{0}$ such that $\frac{1}{n_{0}}<c-c^{\prime}$. Then for every $n \geq n_{0}$, define

$$
C_{n}=\left\{x: f(x) \leq c^{\prime}+\frac{1}{n}\right\}
$$

Those are bounded non empty convex sets (because $f$ is quasi-convex) such that $C_{n+1} \subset C_{n}$ and by the above proposition we get that $\bigcap_{n=n_{0}}^{\infty} C_{n} \neq \emptyset$ and for $x \in \bigcap_{n=n_{0}}^{\infty} C_{n}$ we get that $f(x)=c^{\prime}$. If $f$ is strictly quasi-convex then for every two $x, y \in Z$ such that $f(x)=f(y)=c^{\prime}$ we get that if $x \neq y$ then

$$
f\left(\frac{1}{2} x+\frac{1}{2} y\right)<\max \{f(x), f(y)\}=c^{\prime}
$$

which is a contradiction of the definition of $c^{\prime}$ as the infimum. 
Definition 2.10. A uniquely geodesic metric space $(Z, d)$ is said to be non positively curved in the sense of Busemann, if for every three points: $x, y, z \in Z$ one has

$$
d\left(\frac{1}{2} x+\frac{1}{2} z, \frac{1}{2} y+\frac{1}{2} z\right) \leq \frac{1}{2} d(x, y)
$$

Remark 2.11. Some papers use the term hyperbolic for metric spaces that are non positively curved in the sense of Busemann (see [RS90][Definition 2.1]). We use the term "non positively curved in the sense of Busemann" to avoid confusion with other non equivalent terms as Gromov hyerbolic metric spaces.

Observe that the condition stated it the definition above is equivalent to the condition:

$$
\forall x, y, x^{\prime}, y^{\prime} \in Z, d\left(\frac{1}{2} x+\frac{1}{2} y, \frac{1}{2} x^{\prime}+\frac{1}{2} y^{\prime}\right) \leq \frac{1}{2} d\left(x, y^{\prime}\right)+\frac{1}{2} d\left(x^{\prime}, y\right)
$$

Proposition 2.12. If $(Z, d)$ uniquely geodesic metric space which is non positively curved in the sense of Busemann and uniformly convex, then for every $y \in Z$, the function $d(., y): Z \rightarrow \mathbb{R}$ is a convex function.

Proof. Let $y, x_{1}, x_{2} \in Z$, we need to show that

$$
d\left(\frac{x_{1}+x_{2}}{2}, y\right) \leq \frac{1}{2} d\left(x_{1}, y\right)+\frac{1}{2} d\left(x_{2}, y\right)
$$

Assume WLOG that $d\left(x_{1}, y\right) \geq d\left(x_{2}, y\right)$, then on the geodesic connecting $y$ and $x_{1}$ there is a point $x_{1}^{\prime}$ s.t. $d\left(x_{1}^{\prime}, y\right)=d\left(x_{2}, y\right)$. From uniform convexity we get that

$$
d\left(\frac{x_{1}^{\prime}+x_{2}}{2}, y\right) \leq d\left(x_{2}, y\right)
$$

(Note this need not be a strict inequality because we might have $x_{1}^{\prime}=x_{2}$ ).

From the non positive curvature we get that

$$
d\left(\frac{x_{1}^{\prime}+x_{2}}{2}, \frac{x_{1}+x_{2}}{2}\right) \leq \frac{1}{2} d\left(x_{1}, x_{1}^{\prime}\right)=\frac{1}{2}\left(d\left(x_{1}, y\right)-d\left(x_{2}, y\right)\right)
$$

Therefore

$$
\begin{aligned}
& d\left(\frac{x_{1}+x_{2}}{2}, y\right) \leq d\left(\frac{x_{1}^{\prime}+x_{2}}{2}, y\right)+d\left(\frac{x_{1}^{\prime}+x_{2}}{2}, \frac{x_{1}+x_{2}}{2}\right) \leq \\
\leq & d\left(x_{2}, y\right)+\frac{1}{2}\left(d\left(x_{1}, y\right)-d\left(x_{2}, y\right)\right)=\frac{1}{2} d\left(x_{1}, y\right)+\frac{1}{2} d\left(x_{2}, y\right)
\end{aligned}
$$

Last, observe that if $(Z, d)$ is uniquely geodesic, then for every isometry $T$ of $Z$ and for every two points $x, y \in Z$, one has

$$
T\left(\frac{1}{2} x+\frac{1}{2} y\right)=\frac{1}{2} T(x)+\frac{1}{2} T(y)
$$




\subsection{Reflexive Banach spaces}

In this subsection we will recall some facts about reflexive Banach spaces which will be very similar to the facts we recalled in the previous section.

Proposition 2.13. Let $(Z,||$.$) be a reflexive Banach space, then for closed$ bounded non empty sets $C_{n} \subset Z$ such that $C_{n+1} \subset C_{n}$, we have $\bigcap_{n=1}^{\infty} C_{n} \neq \emptyset$.

Proof. Every set $C_{n}$ is closed and bounded and therefore is compact in the weak topology (because $Z$ is reflexive) and therefore $\bigcap_{n=1}^{\infty} C_{n} \neq \emptyset$.

As in the previous subsection we get the following corollary (the proof is exactly the same):

Corollary 2.14. Let $(Z,||$.$) be a reflexive Banach space and let f: Z \rightarrow \mathbb{R}^{+}$be a quasi-convex function, i.e.

$$
\forall 0 \leq t \leq 1, \forall x, y \in Z, f(t x+(1-t) y) \leq \max \{f(x), f(y)\}
$$

If there is a $c \in \mathbb{R}^{+}$such that the set $\{x: f(x) \leq c\}$ is non empty and bounded, then $f$ has a minimum. Moreover, if $f$ is strictly quasi-convex, i.e.

$$
\forall x, y \in Z, x \neq y, f\left(\frac{1}{2} x+\frac{1}{2} y\right)<\max \{f(x), f(y)\}
$$

then this minimum is unique.

Also observe that for every Banach space $(Z,||$.$) we have$

$$
\forall x, y, x^{\prime}, y^{\prime} \in Z, d\left(\frac{1}{2} x+\frac{1}{2} y, \frac{1}{2} x^{\prime}+\frac{1}{2} y^{\prime}\right) \leq \frac{1}{2} d\left(x, y^{\prime}\right)+\frac{1}{2} d\left(x^{\prime}, y\right)
$$

(Where $d$ is the usual metric induced by the norm) and that for every $y \in Z$, $d(., y): Z \rightarrow \mathbb{R}$ is a convex function.

Finally, recall the Mazur-Ulam theorem (see for instance [FJ03]):

Theorem 2.15. Every surjective isometery between normed spaces is affine.

Which yield that for every isometry $T$ of $(Z,||$.$) and for any two points$ $x, y \in Z$ we have

$$
T\left(\frac{1}{2} x+\frac{1}{2} y\right)=\frac{1}{2} T(x)+\frac{1}{2} T(y)
$$

\subsection{Uniformly convex Busemann non positively curved spaces and Reflexive Banach spaces concluded}

In this subsection we conclude the mutual facts gathered in the last two subsections: let $(Z, d)$ be a uniformly convex, uniquely geodesic, Busemann non positively curved, complete metric space or a reflexive Banach space (where $d$ is the metric induced by the norm), then the following holds: 
- For every strictly convex function $f: Z \rightarrow \mathbb{R}^{+}$if there is a $c \in \mathbb{R}^{+}$such that the set $\{x: f(x) \leq c\}$ is non empty and bounded, then $f$ has a unique minimum.

$$
\forall x, y, x^{\prime}, y^{\prime} \in Z, d\left(\frac{1}{2} x+\frac{1}{2} y, \frac{1}{2} x^{\prime}+\frac{1}{2} y^{\prime}\right) \leq \frac{1}{2} d\left(x, y^{\prime}\right)+\frac{1}{2} d\left(x^{\prime}, y\right)
$$

- For every $y \in Z, d(., y): Z \rightarrow \mathbb{R}$ is a convex function.

- For every isometry $T$ on $Z$ and for every two points $x, y \in Z$, we have

$$
T\left(\frac{1}{2} x+\frac{1}{2} y\right)=\frac{1}{2} T(x)+\frac{1}{2} T(y)
$$

Note that the summation symbols means two different things: in the uniquely geodesic metric space, $\frac{1}{2} x+\frac{1}{2} y$ means the unique midpoint between $x$ and $y$ and

in the Banach case $\frac{1}{2} x+\frac{1}{2} y$ means the average of the two vectors (which is a midpoint, but it need not be unique). From now on we will consider $(Z, d)$ to be either uniformly convex, uniquely geodesic, Busemann non positively curved, complete metric space or a reflexive Banach space and we will use only the mutual facts stated above (and the completeness).

\section{$3 \quad$ Fixed point criteria via links}

Let $\Gamma$ be a group acting on an $n$-dimentional simplicial complex as above, let $(Z, d)$ be either uniformly convex, uniquely geodesic, Busemann non positively curved, complete metric space or a reflexive Banach space and let $\rho: \Gamma \rightarrow$ $\operatorname{Isom}(Z)$. Fix a function $f: \mathbb{R}^{+} \rightarrow \mathbb{R}^{+}$with the following properties:

- $f(0)=0$ and $f$ is strictly monotone increasing.

- $f$ is strictly convex (and therefore $\lim _{x \rightarrow \infty} f(x)=\infty$ ).

- For every constants $0 \leq \kappa<1, C \geq 0$ we have $\sum_{k=1}^{\infty} f^{-1}\left(C \kappa^{k}\right)<\infty$

Examples for such functions are $f(x)=x^{p}$ with $p>1$ and $f(x)=a_{2} x^{2}+\ldots+$ $a_{k} x^{k}$ with $a_{2}, \ldots, a_{k}$ positive.

For every vertex $u \in X$ denote

$$
C^{0}\left(X_{u}\right)=\left\{\phi: \Sigma_{u}(0) \rightarrow Z\right\}
$$

and define $E_{u, \phi}: Z \rightarrow \mathbb{R}^{+}$as

$$
E_{u, \phi}(\xi)=\sum_{v \in \Sigma_{u}(0)} m((u, v)) f(d(\xi, \phi(v)))
$$


Note that $E_{u, \phi}$ is strictly convex, because $f$ is strictly convex and $d(., \phi(v))$ is convex for every $v$. Since $E_{u, \phi}(\xi) \rightarrow \infty$ as $d(\operatorname{Im}(\phi), \xi) \rightarrow \infty$ we get that $E_{u, \phi}$ has a unique minimum. Therefore there is a map $M_{u}: C^{0}\left(X_{u}(0)\right) \rightarrow Z$ which send each $\phi$ to the minimum of $E_{u, \phi}$.

For every $\phi \in C^{0}\left(X_{u}\right)$ define $d_{u} \phi: \Sigma_{u}(1) \rightarrow \mathbb{R}$ to be $d_{u} \phi(v, w)=d(\phi(v), \phi(w))$.

Define for every vertex $u \in X$ a constant $\lambda_{u}$ as following:

$$
\lambda_{u}=\sup \left\{\lambda: \lambda E_{u, \phi}\left(M_{u} \phi\right) \leq \sum_{\eta \in \Sigma_{u}(1)} \frac{m_{u}(\eta)}{2} f\left(d_{u} \phi(\eta)\right), \forall \phi \in C^{0}\left(X_{u}\right)\right\}
$$

Denote

$$
C^{0}(X, \rho)=\{\phi: \Sigma(0) \rightarrow X: \phi \text { is equivariant w.r.t } \rho\}
$$

Define an operator $M: C^{0}(X, \rho) \rightarrow C^{0}(X, \rho)$ as

$$
\forall u \in \Sigma(0), M \phi(u)=\left.M_{u} \phi\right|_{X_{u}}
$$

Where $\left.\phi\right|_{X_{u}}$ is the restriction of $\phi$ to the link of $u$.

For $\phi, \phi^{\prime} \in C^{0}(X, \rho)$ define $\frac{1}{2} \phi+\frac{1}{2} \phi^{\prime} \in C^{0}(X, \rho)$ as

$$
\forall u \in \Sigma(0),\left(\frac{1}{2} \phi+\frac{1}{2} \phi^{\prime}\right)(u)=\frac{1}{2} \phi(u)+\frac{1}{2} \phi^{\prime}(u)
$$

Define the operator $M^{\prime}: C^{0}(X, \rho) \rightarrow C^{0}(X, \rho)$ as

$$
M^{\prime} \phi=\frac{1}{2} \phi+\frac{1}{2} M \phi
$$

Proposition 3.1. The images of the operators $M$ and $M^{\prime}$ are indeed contained in $C^{0}(X, \rho)$.

Proof. To show that the image of $M$ is contained in $C^{0}(X, \rho)$, we need to show that for every $\phi \in C^{0}(X, \rho)$ we have that $M \phi$ is an equivariant map w.r.t $\rho$, i.e. for every $u \in \Sigma(0)$ and every $\gamma \in \Gamma$ we have that

$$
\rho(\gamma) \cdot M \phi(u)=M \phi(\gamma \cdot u)
$$

Fix some $u \in \Sigma(0)$ and $\gamma \in \Gamma$, then $\gamma$ take the link of $u$ to the link of $\gamma \cdot u$ and since $\phi$ is equivariant and the weight $m$ is invariant we get that

$$
\begin{gathered}
E_{\gamma \cdot u,\left.\phi\right|_{X_{\gamma \cdot u}}}(\rho(\gamma) \cdot \xi)=\sum_{\gamma \cdot v \in \Sigma_{\gamma \cdot u}(0)} m((\gamma \cdot u, \gamma \cdot v)) f\left(d\left(\rho(\gamma) \cdot \xi,\left.\phi\right|_{X_{\gamma \cdot u}}(\gamma \cdot v)\right)\right)= \\
=\sum_{\gamma \cdot v \in \Sigma_{\gamma \cdot u}(0)} m((u, v)) f\left(d\left(\rho(\gamma) \cdot \xi,\left.\rho(\gamma) \cdot \phi\right|_{X_{u}}(v)\right)\right)= \\
=\sum_{\gamma \cdot v \in \Sigma_{\gamma \cdot u}(0)} m((u, v)) f(d(\xi, \phi(v)))=E_{u, \phi}(\xi)
\end{gathered}
$$


and therefore if $\left.M_{u} \phi\right|_{X_{u}}$ is the unique minimum of $E_{u,\left.\phi\right|_{X}}(\xi)$ then $\left.\rho(\gamma) \cdot M_{u} \phi\right|_{X_{u}}$ is the unique minimum of $E_{\gamma \cdot u,\left.\phi\right|_{X_{\gamma . u}}}(\xi)$ and the map $M$ is equivariant.

To show $M^{\prime}$ is equivariant we simply recall that for every isometry $T$ of $Z$ and any $x, y \in Z$, we have

$$
T\left(\frac{1}{2} x+\frac{1}{2} y\right)=\frac{1}{2} T(x)+\frac{1}{2} T(y)
$$

Define $E(.,):. C^{0}(X, \rho) \times C^{0}(X, \rho) \rightarrow \mathbb{R}$ as

$$
E(\phi, \psi)=\sum_{(u, v) \in \Sigma(1, \Gamma)} \frac{m((u, v))}{\left|\Gamma_{(u, v)}\right|} f(d(\phi(u), \psi(v)))
$$

Proposition 3.2. 1. For every $\phi, \psi \in C^{0}(X, \rho)$ we have

$$
E(\phi, \psi)=\sum_{u \in \Sigma(0, \Gamma)} \frac{1}{\left|\Gamma_{u}\right|} E_{u, \phi}(\psi(u))
$$

2. For every $\phi \in C^{0}(X, \rho)$ we have

$$
\frac{C(m)}{2} E(\phi, \phi)=\sum_{u \in \Sigma(0, \Gamma)} \frac{1}{\left|\Gamma_{u}\right|} \sum_{\eta \in \Sigma_{u}(1)} \frac{m_{u}(\eta)}{2} f\left(d_{u} \phi(\eta)\right)
$$

Where $C(m)$ is the constant such that for every $\eta \in \Sigma(1)$ we have

$$
\sum_{\sigma \in \Sigma(2), \eta \subset \sigma} m(\sigma)=3 ! C(m) m(\eta)
$$

3. For every $\phi, \phi^{\prime}, \psi, \psi^{\prime} \in C^{0}(X, \rho)$ we have

$$
E\left(\frac{1}{2} \phi+\frac{1}{2} \phi^{\prime}, \frac{1}{2} \psi+\frac{1}{2} \psi^{\prime}\right) \leq \frac{1}{2} E\left(\phi, \psi^{\prime}\right)+\frac{1}{2} E\left(\phi^{\prime}, \psi\right)
$$

Proof. 1. For every $\phi, \psi \in C^{0}(X, \rho)$ we have

$$
\begin{gathered}
\sum_{u \in \Sigma(0, \Gamma)} \frac{1}{\left|\Gamma_{u}\right|} E_{u, \phi}(\psi(u))= \\
=\sum_{u \in \Sigma(0, \Gamma)} \frac{1}{\left|\Gamma_{u}\right|} \sum_{v \in \Sigma_{u}(0)} m((u, v)) f(d(\psi(u), \phi(v)))= \\
=\sum_{u \in \Sigma(0, \Gamma)} \frac{1}{\left|\Gamma_{u}\right|} \sum_{\eta \in \Sigma(1), u \subset \eta} \frac{m(\eta)}{2} f(d(\psi(u), \phi(\eta-u)))
\end{gathered}
$$


Where $\eta-u$ is $v$ for $\eta=(u, v)$ or for $\eta=(v, u)$ (hence the division by 2). Changing the order of summation gives:

$$
\begin{gathered}
\sum_{\eta \in \Sigma(1, \Gamma)} \frac{m(\eta)}{2\left|\Gamma_{\eta}\right|} \sum_{u \in \Sigma(0), u \subset \eta} f(d(\psi(u), \phi(\eta-u)))= \\
=\sum_{(u, v) \in \Sigma(1, \Gamma)} \frac{m((u, v))}{2\left|\Gamma_{(u, v)}\right|}(f(d(\psi(u), \phi(v)))+f(d(\psi(v), \phi(u)))= \\
=\sum_{(u, v) \in \Sigma(1, \Gamma)} \frac{m((u, v))}{\left|\Gamma_{(u, v)}\right|} f(d(\psi(u), \phi(v)))=E(\phi, \psi)
\end{gathered}
$$

2. For every $\phi \in C^{0}(X, \rho)$ we have

$$
\begin{gathered}
\sum_{u \in \Sigma(0, \Gamma)} \frac{1}{\left|\Gamma_{u}\right|} \sum_{\eta \in \Sigma_{u}(1)} \frac{m_{u}(\eta)}{2} f\left(d_{u} \phi(\eta)\right)= \\
\sum_{u \in \Sigma(0, \Gamma)} \frac{1}{\left|\Gamma_{u}\right|} \sum_{\sigma \in \Sigma(2), u \subset \sigma} \frac{m(\sigma)}{6} f\left(d_{u} \phi(\sigma-u)\right)
\end{gathered}
$$

Where again $\sigma-u=(v, w)$ for $\sigma=(u, v, w),(v, u, w),(v, w, u)$. Changing the order of summation gives

$$
\begin{gathered}
\sum_{\sigma \in \Sigma(2, \Gamma)} \frac{m(\sigma)}{6\left|\Gamma_{\sigma}\right|} \sum_{u \in \Sigma(0), u \subset \sigma} f\left(d_{u} \phi(\sigma-u)\right)= \\
\sum_{\sigma \in \Sigma(2, \Gamma)} \frac{m(\sigma)}{6\left|\Gamma_{\sigma}\right|} \sum_{\eta \in \Sigma(1), \eta \subset \sigma} \frac{1}{2} f(d \phi(\eta))
\end{gathered}
$$

Where $d \phi((v, w))=d\left(\phi(v), \phi(w)\right.$ and the factor $\frac{1}{2}$ is because $(v, w),(w, v) \subset$ $(u, v, w)$. Again we can change the order of summation and get

$$
\begin{gathered}
\sum_{\eta \in \Sigma(1, \Gamma)} \frac{f(d \phi(\eta))}{12\left|\Gamma_{\eta}\right|} \sum_{\sigma \in \Sigma(2), \eta \subset \sigma} m(\sigma)= \\
\sum_{\eta \in \Sigma(1, \Gamma)} \frac{C(m) m(\eta) f(d \phi(\eta))}{2\left|\Gamma_{\eta}\right|}=\frac{C(m)}{2} E(\phi, \phi)
\end{gathered}
$$

3. For every $\phi, \phi^{\prime}, \psi, \psi^{\prime} \in C^{0}(X, \rho)$ we have

$$
\begin{gathered}
E\left(\frac{1}{2} \phi+\frac{1}{2} \phi^{\prime}, \frac{1}{2} \psi+\frac{1}{2} \psi^{\prime}\right)=\sum_{(u, v) \in \Sigma(1, \Gamma)} \frac{m((u, v))}{\left|\Gamma_{(u, v)}\right|} f\left(d\left(\frac{1}{2} \phi(u)+\frac{1}{2} \phi^{\prime}(u), \frac{1}{2} \psi(v)+\frac{1}{2} \psi^{\prime}(v)\right)\right) \leq \\
\leq \sum_{(u, v) \in \Sigma(1, \Gamma)} \frac{m((u, v))}{\left|\Gamma_{(u, v)}\right|} f\left(\frac{1}{2} d\left(\phi(u), \psi^{\prime}(v)\right)+\frac{1}{2} d\left(\phi^{\prime}(u), \psi(v)\right)\right)
\end{gathered}
$$


Where the inequality follows for properties of $Z$ and for the fact that $f$ is monotone increasing. From convexity of $f$ we get

$$
\begin{gathered}
\leq \sum_{(u, v) \in \Sigma(1, \Gamma)} \frac{m((u, v))}{\left|\Gamma_{(u, v)}\right|}\left(\frac{1}{2} f\left(d\left(\phi(u), \psi^{\prime}(v)\right)+\frac{1}{2} f\left(d\left(\phi^{\prime}(u), \psi(v)\right)\right)\right)=\right. \\
\frac{1}{2} E\left(\phi, \psi^{\prime}\right)+\frac{1}{2} E\left(\phi^{\prime}, \psi\right)
\end{gathered}
$$

Denote $\lambda=\min \left\{\lambda_{u}: u \in \Sigma(0)\right\}$, then from the above proposition we get:

Corollary 3.3. 1. For every $\phi \in C^{0}(X, \rho)$ we have that

$$
\frac{C(m)}{2} E(\phi, \phi) \geq \lambda E(M \phi, \phi)
$$

2. For every $\phi \in C^{0}(X, \rho)$ we have that

$$
\frac{C(m)}{2} E(\phi, \phi) \geq \lambda E\left(M^{\prime} \phi, M^{\prime} \phi\right)
$$

Proof. 1. For every $\phi \in C^{0}(X, \rho)$, by 2 . in the above proposition we get that

$$
\begin{gathered}
\frac{C(m)}{2} E(\phi, \phi)=\sum_{u \in \Sigma(0, \Gamma)} \frac{1}{\left|\Gamma_{u}\right|} \sum_{\eta \in \Sigma_{u}(1)} \frac{m_{u}(\eta)}{2} f\left(d_{u} \phi(\eta)\right) \geq \\
\geq \lambda \sum_{u \in \Sigma(0, \Gamma)} \frac{1}{\left|\Gamma_{u}\right|} E_{u, \phi}\left(M_{u} \phi\right) \geq \lambda E(\phi, M \phi)
\end{gathered}
$$

Where the first inequality is due to the definition of $\lambda$ and the second inequality is due to 1 . in the above proposition.

2. Due to 3 . in the above proposition we get that

$$
\begin{aligned}
& \lambda E\left(M^{\prime} \phi, M^{\prime} \phi\right)=\lambda E\left(\frac{1}{2} \phi+\frac{1}{2} M \phi, \frac{1}{2} \phi+\frac{1}{2} M \phi\right) \leq \\
& \leq \frac{1}{2} \lambda E(\phi, M \phi)+\frac{1}{2} \lambda E(M \phi, \phi) \leq \frac{C(m)}{2} E(\phi, \phi)
\end{aligned}
$$

Theorem 3.4. If $\lambda>\frac{C(m)}{2}$ then $\Gamma$ has a fixed point for every $\rho$. 
Proof. Denote by $\kappa=\frac{C(m)}{2 \lambda}$, then $0 \leq \kappa<1$ and for every $\phi \in C^{0}(X, \rho)$

$$
\kappa E(\phi, \phi) \geq E\left(M^{\prime} \phi, M^{\prime} \phi\right)
$$

Therefore, for every $k \in \mathbb{N}$ we have

$$
\kappa^{k} E(\phi, \phi) \geq E\left(\left(M^{\prime}\right)^{k} \phi,\left(M^{\prime}\right)^{k} \phi\right)
$$

Denote by $\delta=\min \{m(\eta): \eta \in \Sigma(1)\}>0$, then for every $(u, v) \in \Sigma(1, \Gamma)$ we have

$$
\kappa^{k} E(\phi, \phi) \geq E\left(\left(M^{\prime}\right)^{k} \phi,\left(M^{\prime}\right)^{k} \phi\right) \geq \delta f\left(d\left(\left(M^{\prime}\right)^{k} \phi(u), d\left(\left(M^{\prime}\right)^{k} \phi(v)\right)\right)\right.
$$

and therefore, for every $(u, v) \in \Sigma(1, \Gamma)$ we have

$$
f^{-1}\left(\frac{\kappa^{k} E(\phi, \phi)}{\delta}\right) \geq d\left(\left(M^{\prime}\right)^{k} \phi(u), d\left(\left(M^{\prime}\right)^{k} \phi(v)\right)\right.
$$

We also have for every $\phi \in C^{0}(X, \rho)$ that

$$
\kappa E(\phi, \phi) \geq E(M \phi, \phi)
$$

and therefore

$$
E\left(\left(M^{\prime}\right)^{k} \phi,\left(M^{\prime}\right)^{k} \phi\right)>\kappa E\left(\left(M^{\prime}\right)^{k} \phi,\left(M^{\prime}\right)^{k} \phi\right) \geq E\left(\left(M^{\prime}\right)^{k} \phi, M\left(M^{\prime}\right)^{k} \phi\right)
$$

So we have

$$
\begin{gathered}
E\left(\left(M^{\prime}\right)^{k} \phi,\left(M^{\prime}\right)^{k+1} \phi\right)=E\left(\left(M^{\prime}\right)^{k} \phi, \frac{1}{2}\left(M^{\prime}\right)^{k} \phi+\frac{1}{2} M\left(M^{\prime}\right)^{k} \phi\right) \leq \\
\leq \frac{1}{2} E\left(\left(M^{\prime}\right)^{k} \phi,\left(M^{\prime}\right)^{k} \phi\right)+\frac{1}{2} E\left(\left(M^{\prime}\right)^{k} \phi, M\left(M^{\prime}\right)^{k} \phi\right)<E\left(\left(M^{\prime}\right)^{k} \phi,\left(M^{\prime}\right)^{k} \phi\right)
\end{gathered}
$$

Therefore, for every $(u, v) \in \Sigma(1, \Gamma)$ we have (as before)

$$
f^{-1}\left(\frac{\kappa^{k} E(\phi, \phi)}{\delta}\right) \geq d\left(\left(M^{\prime}\right)^{k} \phi(u),\left(M^{\prime}\right)^{k+1} \phi(v)\right)
$$

By triangle inequality we have for every $u \in \Sigma(0)$

$$
2 f^{-1}\left(\frac{\kappa^{k} E(\phi, \phi)}{\delta}\right) \geq d\left(\left(M^{\prime}\right)^{k} \phi(u),\left(M^{\prime}\right)^{k+1} \phi(u)\right)
$$

Now since

$$
\sum_{k=1}^{\infty} 2 f^{-1}\left(\frac{\kappa^{k} E(\phi, \phi)}{\delta}\right)<\infty
$$

Then for every $u \in \Sigma(0),\left(M^{\prime}\right)^{k} \phi(u)$ is Cauchy sequence and therefore we can define $\phi_{0} \in C^{0}(X, \rho)$ as

$$
\forall u \in \Sigma(0), \phi_{0}(u)=\lim \left(M^{\prime}\right)^{k} \phi(u)
$$

We get that $E\left(\phi_{0}, \phi_{0}\right)=0$ and therefore $\phi_{0}$ must be a constant, equivariant map (so there is a fixed point). 
Corollary 3.5. The above theorem generalizes several previous theorems:

1. For the case $f(x)=x^{2}$ and $Z$ is a Hilbert space, we get the criterion stated in [BŚ97] and [Żuk96] (this is the famous Żuk criterion).

2. For the case $f(x)=x^{2}$ and $Z$ is a Hadamard space (i.e. CAT(0) and complete), we get the criterion stated in [IN05] (for the 2 dimensional case).

3. For the case $f(x)=x^{p}$ and $Z$ is $L^{p}$ for $1<p<\infty$, we get the criterion in [Bou12] (for the 2 dimensional case).

4. For the case $f(x)=x^{p}$ and $Z$ is a reflexive Banach space, we improve the criterion in [Now].

Remark 3.6. Note that in the case that $Z$ is a Banach space, the reflexivity of $Z$ and the strict convexity of $f$ were only required to define $M$ uniquely and to insure it is an equivariant operator. We can avoid those restrictions and work in a general Banach space, if we define a different equivariant operator M. For instance, if we define

$$
M \phi_{u}=\sum_{v \in \Sigma_{u}(0)} \frac{m_{u}(v)}{\sum_{v \in \Sigma_{u}(0)} m_{u}(v)} \phi(v)
$$

Then be Mazur-Ulam theorem, $M$ will be an equivariant operator and we have the same criterion with the appropriate $\lambda$.

\section{References}

[BdlHV08] Bachir Bekka, Pierre de la Harpe, and Alain Valette. Kazhdan's property (T), volume 11 of New Mathematical Monographs. Cambridge University Press, Cambridge, 2008.

[Bou12] Marc Bourdon. Un théorème de point fixe sur les espaces $L^{p}$. Publ. Mat, 56:375-392, 2012.

[BŚ97] W. Ballmann and J. Świạtkowski. On $L^{2}$-cohomology and property (T) for automorphism groups of polyhedral cell complexes. Geom. Funct. Anal., 7(4):615-645, 1997.

[DJ00] Jan Dymara and Tadeusz Januszkiewicz. New Kazhdan groups. Geom. Dedicata, 80(1-3):311-317, 2000.

[FJ03] Richard J. Fleming and James E. Jamison. Isometries on Banach spaces: function spaces, volume 129 of Chapman $83 \mathrm{Hall} / \mathrm{CRC}$ Monographs and Surveys in Pure and Applied Mathematics. Chapman \& Hall/CRC, Boca Raton, FL, 2003. 
[GKM08] Tsachik Gelander, Anders Karlsson, and Gregory A. Margulis. Superrigidity, generalized harmonic maps and uniformly convex spaces. Geom. Funct. Anal., 17(5):1524-1550, 2008.

[GR84] Kazimierz Goebel and Simeon Reich. Uniform convexity, hyperbolic geometry, and nonexpansive mappings, volume 83 of Monographs and Textbooks in Pure and Applied Mathematics. Marcel Dekker Inc., New York, 1984.

[Gro03] M. Gromov. Random walk in random groups. Geom. Funct. Anal., 13(1):73-146, 2003.

[IN05] Hiroyasu Izeki and Shin Nayatani. Combinatorial harmonic maps and discrete-group actions on Hadamard spaces. Geom. Dedicata, 114:147-188, 2005.

[KL10] U. Kohlenbach and L. Leuştean. Asymptotically nonexpansive mappings in uniformly convex hyperbolic spaces. J. Eur. Math. Soc. (JEMS), 12(1):71-92, 2010.

[Now] Piotr Nowak. Poincaré inequalities and rigidity for actions on banach spaces. Preprint.

[RS90] Simeon Reich and Itai Shafrir. Nonexpansive iterations in hyperbolic spaces. Nonlinear Anal., 15(6):537-558, 1990.

[Żuk96] Andrzej Żuk. La propriété (T) de Kazhdan pour les groupes agissant sur les polyèdres. C. R. Acad. Sci. Paris Sér. I Math., 323(5):453458, 1996. 